\title{
Estudo e Análise de Processamento Paralelo em Simulações de Fluidodinâmica Computacional com OpenFOAM
}

\author{
Valesca Moura de Sousa ${ }^{1}$, Fernanda Gonçalves de Oliveira Passos ${ }^{2}$ \\ ${ }^{1}$ Instituto de Computação - Universidade Federal Fluminense (UFF) - Niterói - RJ \\ ${ }^{2}$ Escola de Engenharia - Universidade Federal Fluminense (UFF) - Niterói - RJ \\ \{valescamoura, fernanda\}@midiacom.uff.br
}

\begin{abstract}
This papers aims to analyze the performance of parallel methods implemented in the computational fluid-dynamic tool, OpenFOAM. This analysis is based on two cases in different contexts and complexities. The results shows that the considered parallel methods of the tool do not scale and specific efforts to adjust input parameters are necessary for convergence in complex cases.
\end{abstract}

Resumo. Este artigo tem o objetivo de analisar o desempenho dos métodos paralelos implementados na ferramenta de fluidodinâmica computacional, OpenFOAM. Esta análise é feita com base em dois casos de contextos e complexidades distintas. Os resultados mostram que os métodos paralelos avaliados da ferramenta não escalam e que é necessário um esforço específico de ajuste de parâmetros para haver convergência em casos complexos e paralelos.

\section{Introdução}

A Fluidodinâmica Computacional (Computational fluid dynamics, CFD) é a análise de sistemas envolvendo escoamento de fluidos, transferência de calor e fenômenos associados [Versteeg and Malalasekera 2007]. Tal análise é de suma importância em diversas áreas, como física e engenharias e o OpenFOAM [OpenFOAM 2019] é uma ferramenta bastante utilizada para este propósito. No entanto, as simulações CFD apresentam cálculos muito custosos mesmo para os computadores potentes que existem atualmente. Nesse contexto, a computação paralela é empregada e o OpenFOAM possui atualmente versões paralelas e distribuídas para melhorar a eficiência de programas CFD.

O objetivo deste trabalho ${ }^{1}$ é analisar o desempenho dos métodos paralelos do OpenFOAM nos quesitos uso de memória e speed-up. Além disso, serão avaliados os resultados numéricos a fim de verificar como o particionamento do problema na implementação paralela influencia os resultados da simulação em relação à execução sequencial. Para tal, são utilizados dois experimentos: um monofásico e outro multifásico.

\section{OpenFOAM e Trabalhos Relacionados}

O OpenFOAM [OpenFOAM 2019] é uma ferramenta CFD de código aberto - motivo pelo qual foi escolhida nesse estudo - implementada em C++. Sua implementação paralela se baseia no particionamento da malha entre os processos de modo a executar o algoritmo serial do solucionador iterativo. A cada iteração, é necessária a comunicação

\footnotetext{
${ }^{1}$ Este trabalho é financiado pelo projeto RAVES/Petrobras, 2020.
} 
entre os processos para trocarem dados de alguns pontos da malha em comum. Para isso, o MPI - Message Passing Interface - é utilizado. Os métodos de particionamento de malha disponíveis no OpenFOAM e analisados neste trabalho são: 1) Simple: decomposição geométrica na qual o domínio é dividido em partes por direção no eixo $x, y$ e $z ; 2)$ Hierarchical: similar ao simple, porém é possível especificar a ordem da divisão direcional; 3) Scotch: busca minimizar o número de limites da malha de cada processo.

Há diversos trabalhos que discutem o paralelismo da ferramenta OpenFOAM. Alguns deles indicam que o gargalo na escalabilidade dos solucionadores OpenFOAM está ligado à massiva comunicação MPI entre processadores relacionada tanto a forma de particionamento da malha [Wang et al. 2012] quanto ao solucionador iterativo selecionado [Rivera and Furlinger 2011]. Nesse contexto, existem autores que propuseram melhorias através de um novo método de particionamento estático a fim de melhorar desempenho e, também, convergência de fluxos multifásicos [Xu et al. 2019]. O trabalho em [Lin et al. 2018] propõe otimizações na comunicação de solucionadores iterativos.

\section{Metodologia da Análise}

Com o intuito de analisar o desempenho do OpenFOAM e a influência de seus métodos paralelos nos resultados numéricos, foram feitos experimentos com múltiplas medições de tempo de execução para cálculo do speed-up. Além disso, foi monitorada a utilização de memória durante as execuções seriais e paralelas. Nas simulações, foram avaliados três métodos paralelos da ferramenta: simple, hierarchical e scotch. Posteriormente, foi feita uma análise dos resultados numéricos da execução paralela para avaliar como o particionamento da malha com o método scotch pode influenciar os resultados da simulação em relação à execução sequencial. Os resultados analisados referem-se aos valores dos campos e, em ambos os casos, tais valores foram extraídos através do singleGraph, utilitário fornecido pela aplicação OpenFOAM, para 3 linhas onde foram observadas maiores movimentações da malha.

Os experimentos foram conduzidos em um cluster composto por 5 nós, cada um com um processador quadcore Intel(R) Core i 7 com memória principal de $32 \mathrm{~GB}$, conectados por rede do tipo Gigabit Ethernet. Os casos de teste utilizados foram o PitzDaily, monofásico e permanente e, DamBreak, multifásico e transiente. As configurações aplicadas na preparação de ambos os casos encontram-se disponíveis no diretório tutorials incluso na instalação do OpenFOAM versão 7. Ademais, as malhas foram refinadas em 16 vezes seus tamanhos originais, a fim de aumentar o processamento.

\section{Resultados}

Para a análise de desempenho, o gráfico da Figura 1 mostra o speed-up obtido conforme aumento do número de processos em uma execução realizada em 1 e 2 nós do cluster. À esquerda temos os valores para o PitzDaily e, à direita, para o DamBreak. De toda forma, percebemos que nenhum dos métodos paralelos utilizados escalam bem. Para o caso bifásico DamBreak, que é mais complexo, os valores de speed-ups foram ainda menores.

Além disso, constatou-se que o uso de memória durante a execução da aplicação não é ideal em nenhum dos casos. Por exemplo, uma execução serial do caso DamBreak utiliza cerca de $125 \mathrm{MB}$. Em uma execução paralela com 2 processos, um dos processos utiliza por volta de $106 \mathrm{MB}$. Portanto, é possível concluir que a execução paralela utiliza mais memória do que a serial. Isso pode levar a um aumento de cache misses. 


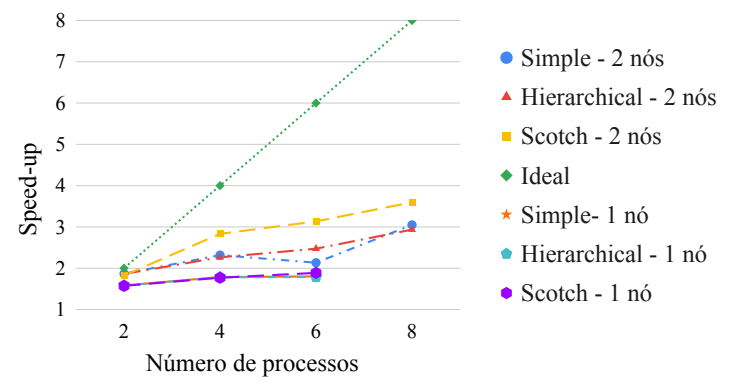

(a) PitzDaily.

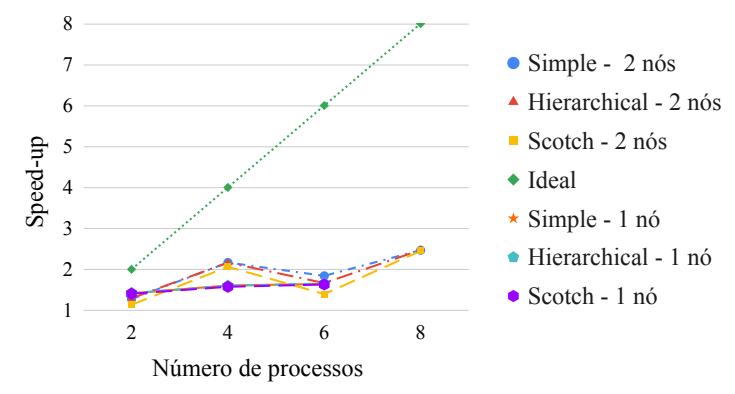

(b) DamBreak.

Figura 1. Speed-up calculados sobre a média de tempos de 30 rodadas.

Para a análise dos resultados numéricos das simulações, observou-se a diferença percentual dos valores obtidos no tempo de simulação dos experimentos em comparação ao valor absoluto numérico para campos como pressão e velocidade. Para tal, comparouse os resultados de uma execução serial e paralela com 4 processos. No caso PitzDaily as diferenças não são representativas o suficiente tendo como maior valor de diferença relativa $0,1 \%$. Em contrapartida, DamBreak apresenta diferenças consideráveis de até $22,9 \%$ em alguns pontos da malha quando comparadas as execuções seriais e paralelas.

\section{Conclusão e trabalhos futuros}

O presente estudo demostrou que os métodos paralelos disponíveis na ferramenta OpenFOAM não apresentam boa escalabilidade. Tal problema pode estar associado à latência de comunicação MPI entre máquinas e à alta taxa de cache misses, que devem ser analisados futuramente. Pontualmente, é possível afirmar que o método scotch apresenta um melhor desempenho que os demais em casos simples, como o PitzDaily.

Em casos complexos, como o DamBreak, o desempenho e corretude numérica de tal método, em relação a simulação serial, não é adequado. Isto mostra que, para casos multifásicos, os parâmetros do método devem ser ajustados especificamente para as execuções paralelas. O estudo destes parâmetros é mais um trabalho futuro.

\section{Referências}

Lin, Z. et al. (2018). Communication optimization for multiphase flow solver in the library of OpenFOAM. Water, 10(10):1461.

OpenFOAM (2019). The open source CFD toolbox. http: / / www . openfoam. com. Acessado em Outubro de 2020.

Rivera, O. and Furlinger, K. (2011). Parallel aspects of openfoam with large eddy simulations. In 2011 IEEE Int. Conf. on HPC and Communications, pages 389-396.

Versteeg, H. K. and Malalasekera, W. (2007). An Introduction to Computational Fluid Dynamics: The Finite Volume Method. Editora Prentice Hall, 2 edition.

Wang, M. et al. (2012). Performance analysis of the graph-partitioning algorithms used in OpenFOAM. IEEE 5th Int. Conf. on Advanced Comp. Intelligence, pages 99-104.

Xu, L., Wang, M., Xu, X., Cui, C., and Yang, X. (2019). Dynamic mesh re-partitioning considering iterative convergence rate for multiphase flows in OpenFOAM. Concurrency and Computation: Practice and Experience, 31(21):e5412. 